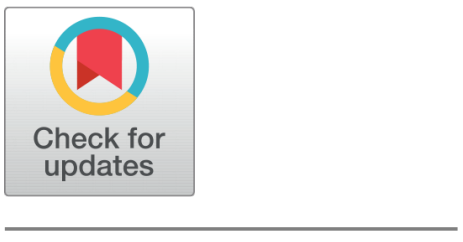

OPEN ACCESS

Received: 07.09.2020

Accepted: 14.12 .2020

Published: 30.12 .2020

Citation: Kumar J, Kumar V, Singh VK, Mittal U, Fahim , Barlewar DS, Nimal AT (2020) GC-SAW and GC-FID performance comparison for fast analysis of volatile organic compounds. Indian Journal of Science and Technology 13(48): 4672-4678. https://doi.org/ 10.17485/IJST/v13i48.1611

* Corresponding author.

vinayksdu@gmail.com

Funding: None

Competing Interests: None

Copyright: (c) 2020 Kumar et al. This is an open access article distributed under the terms of the Creative Commons Attribution License, which permits unrestricted use, distribution, and reproduction in any medium, provided the original author and source are credited.

Published By Indian Society for Education and Environment (iSee)

ISSN

Print: 0974-6846

Electronic: 0974-5645

\section{GC-SAW and GC-FID performance comparison for fast analysis of volatile organic compounds}

\author{
Jitender Kumar ${ }^{1,2}$, Vinod Kumar ${ }^{3}$, Vinay Kumar Singh ${ }^{4 *}$, Upendra Mittal ${ }^{1}$, \\ Fahim ${ }^{1}$, Devendra S Barlewar ${ }^{1}$, A T Nimal ${ }^{1}$ \\ 1 Solid State Physics Laboratory, Delhi, India \\ 2 Department of Chemistry, Delhi University, Delhi, India \\ 3 Special Centre for Nano Sciences, Jawaharlal Nehru University, Delhi, 110067, India \\ 4 Department of Chemistry, Sri Aurobindo College, University of Delhi, Delhi, India
}

\section{Abstract}

Objectives: To develop gas chromatograph (GC) based chemical agent detector and to compare the response of Surface Acoustic Wave (SAW) detector with Flame Ionization Detector (FID) for the development of a reliable and fast gas/vapour analyzer. Also, to describe the limitations of the FID over SAW detector. Methods: An uncoated 433.92 MHz SAW device was used as Gas Chromatography (GC) detector and its response was recorded and compared with conventional FID detector using short capillary column. The response of both the detectors were analyzed by using standard mixture of seven Volatile Organic Compounds (VOCs). Besides this comparison various key parameter of GC i.e. Flow, temperature and length of GC column were also optimized for fast GC analysis. Findings: After analyzing the fast GC data of both the detectors with same sample, it was observed that the resolution of chromatograms with SAW detector showed more resolved peaks as compare to the FID detector for the same GC parameters. Improvements/Applications: It is concluded that the SAW detector is more suitable for fast and reliable analysis of chemical vapors in rapid analysis.

Keywords: Gas chromatograph detector; surface acoustic wave; volatile organic compounds

\section{Introduction}

Surface Acoustic Wave (SAW) device was used in 1979 for the first time as gas sensing and in 1964 as detector ${ }^{(1,2)}$. In order to the detection of VOCs researchers used many technologies i.e. mass spectroscopy, ion mobile spectroscopy etc. ${ }^{(3,4)}$ Gas chromatograph is one of the best techniques used for the analysis of organic volatile compounds employing various detectors. In order to make the fast analysis researcher optimized the various parameters for development of fast gas chromatography ${ }^{(5-9)}$. The sensitivity and resolution are the key parameter of any gas sensor system. Therefore, it becomes of the utmost importance to have a system with the best possible resolution. Mostly for the analysis and identification of toxic gases/vapors in field scenario, the 
Gas chromatography techniques with selective or non-selective detectors is used for the detection of chemical vapors. The Flame ionization (FID), Thermal conductivity (TCD), photo-ionization (PID) etc., are non-selective detectors whereas nitrogen phosphorous (NPD), electron capture (ECD), or mass (MS) are selective detectors. The property of few detectors shown in the Table $1^{(3-6,10,11)}$.

Table 1. Major detector for gas chromatography

\begin{tabular}{lllllll}
\hline Parameter & FID & NPD & FPD & MS & ECD & SAW \\
\hline Sensitivity(g/s) & $2 \times 10^{-12}$ & $2 \times 10^{-13}$ & $1 \times 10^{-12}$ & $1 \times 10^{-9}$ & $5 \times 10^{-14}$ & $1 \times 10^{-15}$ \\
Dynamic Range & $1 \times 10^{7}$ & $1 \times 10^{5}$ & $1 \times 10^{5}$ & $1 \times 10^{6}$ & $1 \times 10^{2}$ & $1 \times 10^{7}$ \\
Selectivity & No & Yes & Yes & Yes & Yes & No \\
\hline
\end{tabular}

SAW detector used as Non-selective GC detector shows the linear response towards the mass change over the surface of the detector ${ }^{(6)}$. To the best of our knowledge, in literature no comparative study of resolution considering two different detectors (SAW/FID) done earlier for fast analysis.

In this study, we are presenting how the resolution of detectors under study is affected with variation of gas chromatographic parameters. Different detector needs different types of gases for the operation as per the detector requirement like FID needs Hydrogen and Zero Air in the ratio of 1:10. However, the SAW detector does not require any specific gases for the operation.

\section{SAW detector operation}

A SAW device consists of a piezoelectric substrate with two transducers called inter digital transducers (IDT) input and output IDTs. On applying the RF signal on input IDTs, it generates the surface acoustic wave on the surface of device and vice versa happened on the output IDT ${ }^{(11-15)}$. A schematic of SAW detector shown in Figure 1. It works on the principle of mass loading, when the vapour molecules are eluted from the capillary column and adsorbed on the surface of detector hence SAW velocity changes and it reflected as the change in resonance frequency of SAW detector. This relationship had been represented by Sauerbrey equation $(1)^{(15)}$.

$$
\Delta f=-\frac{2 f_{0}^{2}}{A \sqrt{\rho_{q} \mu_{q}}} \Delta \mathrm{m}
$$

Where $\Delta \mathrm{f}$ - frequency change, $\mathrm{f}_{0}$ - resonant frequency, $\Delta \mathrm{m}$ - mass change $(\mathrm{g}), \mathrm{A}$ - active crystal area, $\rho_{q}$ - density of substrate material and $\mu \mathrm{q}$ - Shear modulus of substrate.

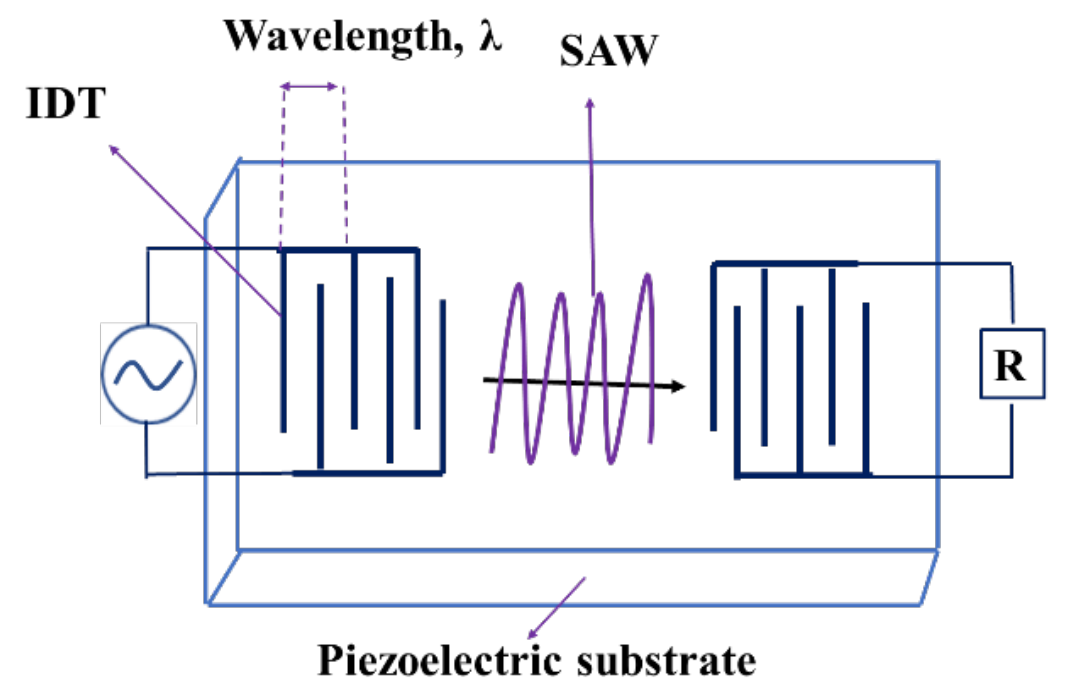

Fig 1. Schematic of SAW device 


\section{FID detector operation}

The FID is a destructive technique in which the sample breaks into ions. The FID response is based upon the concentration of ions which were formed during the combustion of organic compounds. The same ions collected on the electrode and produce an electrical signal which consider as the detector response. This detector needs appropriate mixture of Zero air and hydrogen gas for operation ${ }^{(16,17)}$. When the mixture of compounds eluted from the GC column and passes through the flame, it produces ions as follows-

$$
\text { Organic sample }+\mathrm{O}_{2}->\mathrm{C}^{+}+\mathrm{H}_{2} \mathrm{O}+\mathrm{e}-->\mathrm{CO}_{2}+\mathrm{H}_{2} \mathrm{O}
$$

This detector is used for the detection of those sample which are hydrocarbons and having C-H bonds. Hydrogen gas are used as fuel gas for operation of FID. Schematic of FID is shown in Figure 2 .

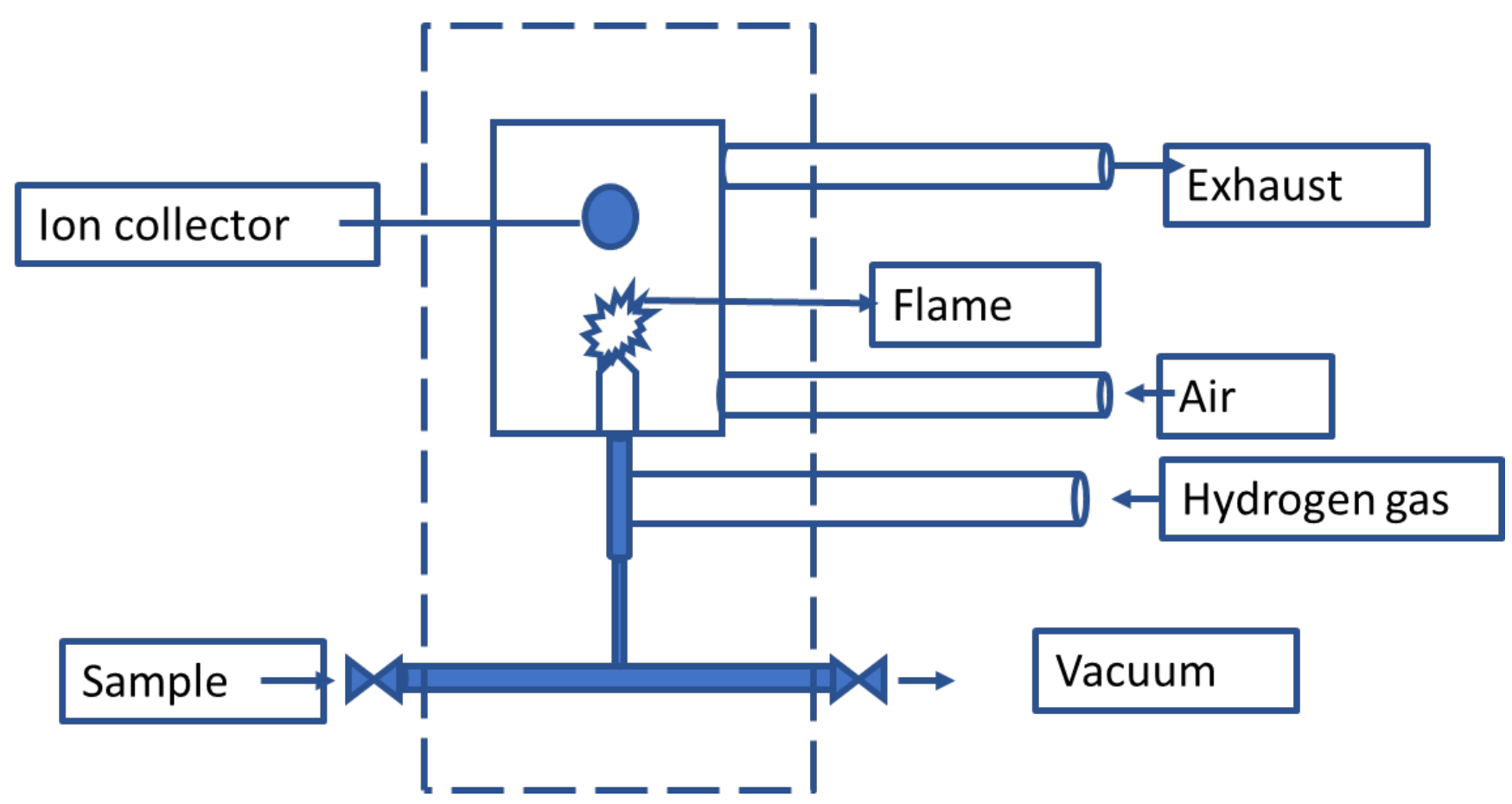

Fig 2. Schematic of FID

\section{Gas chromatography (GC)}

GC technique is used to separates the mixture of different compounds between mobile and stationary phase of column (capillary/packed) and detects by the selected detector as electronic signal over a period called retention time and time-resolved chemical spectra known as chromatogram. Different chemical constituents of the sample travel through the column at different rates depending upon their physical and chemical properties, the interaction of sample will be different with the phases (mobile and stationary). In order to achieve, the non-overlapping peaks of compounds the GC parameters i.e. length of column, flow of carrier gas, temperature etc. has to be optimized. The eluted vapors of samples from the column are detected by Detector ${ }^{(10,11)}$.

\section{Experimental}

For performing the experimental work, a conventional GC with FID detector (Make chemito-1000) system was used. A 433.92 $\mathrm{MHz}$ one-port SAW oscillator used as a detector (Figure 3 ) which has two identical SAW device, one act as detector and another as a reference. A small Thermoelectric cooler (TEC) (Size - 12.3×12.3 mm, (make Laird Technologies, part No. CP0.8-3106L) was used to maintain the SAW detector temperature at room temperature. The temperature of assembled SAW detector is measured by PT1000 RTD. The SAW detector enclosure assembly including TEC, heat sink and sensor cell are shown in Figure 3. 
The detector cell has the provision for connecting the capillary column to the SAW detector (Figure 3). It has been designed specifically for exposing only one SAW device. The detector response of SAW detector is considered in terms of frequency shift.

For entire experiment Rtx-200 narrow bore capillary column was used with $0.2 \mu \mathrm{l}$ sample. The injector port was kept at $130^{\circ} \mathrm{C}$. A standard mixture was prepared by mixing the entire 7 analytical grade VOCs in equal ratio.

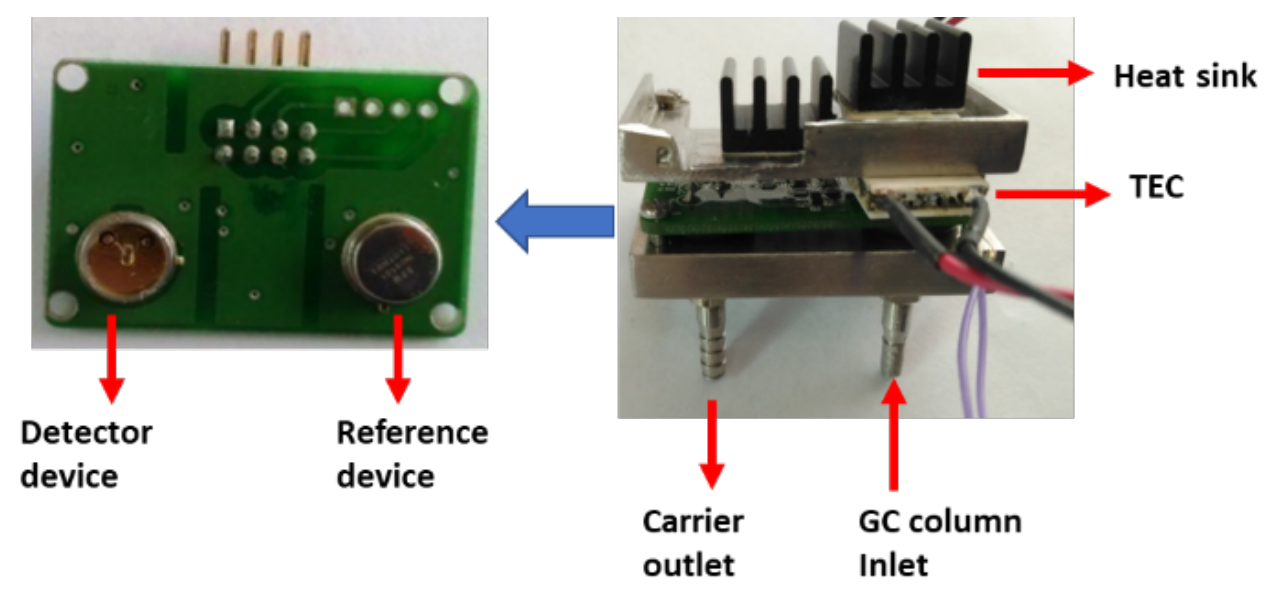

Fig 3. SAW detector

The responses of both detectors were recorded at various parameters of GC. Initially $30 \mathrm{~m}, 15 \mathrm{~m}, 7 \mathrm{~m}$, and $3.75 \mathrm{~m}$ meters capillary column were used at various oven temperature and carrier flow rate using FID detector. Since the chromatogram with $7 \mathrm{~m}$ column was well resolved whereas the chromatogram became unresolved at $3.75 \mathrm{~m}$. Therefore, the $3.75 \mathrm{~m}$ column was selected for comparing the chromatogram of mixture of compounds with SAW and FID detector.

\section{Result and Discussion}

A mixture of seven compounds (Methanol, Toluene, Xylene, Dimethylformamide (DMF), 1,5-Dichloropentane (DCP), Dibutyl sulfide (DBS) and Nitrobenzene (NB)) were used for the injection in conventional GC system with FID detector as well as SAW detector. The responses of both the detectors were measured with different parameters of GC system. The retention time of nitrobenzene was recorded with different flow rate of the carrier gas (nitrogen gas) and found that at higher flow rate of carrier gas the resolution of chromatogram decreases (Figure 4 ).

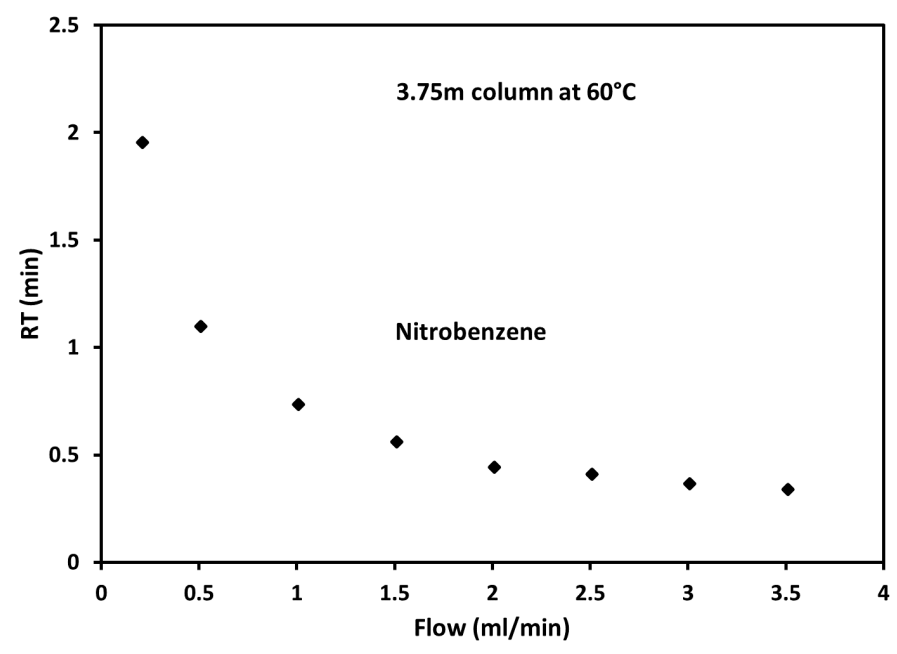

Fig 4. Effect of carrier flow on Retention time of NB 
Similarly, at $60^{\circ} \mathrm{C}$ the Nitrobenzene (NB) and Dibutyl sulfide (DBS) response was recorded at various length of the capillary column and found that the retention time drastically reduced with decreasing the length of capillary column (Figure 5 ).

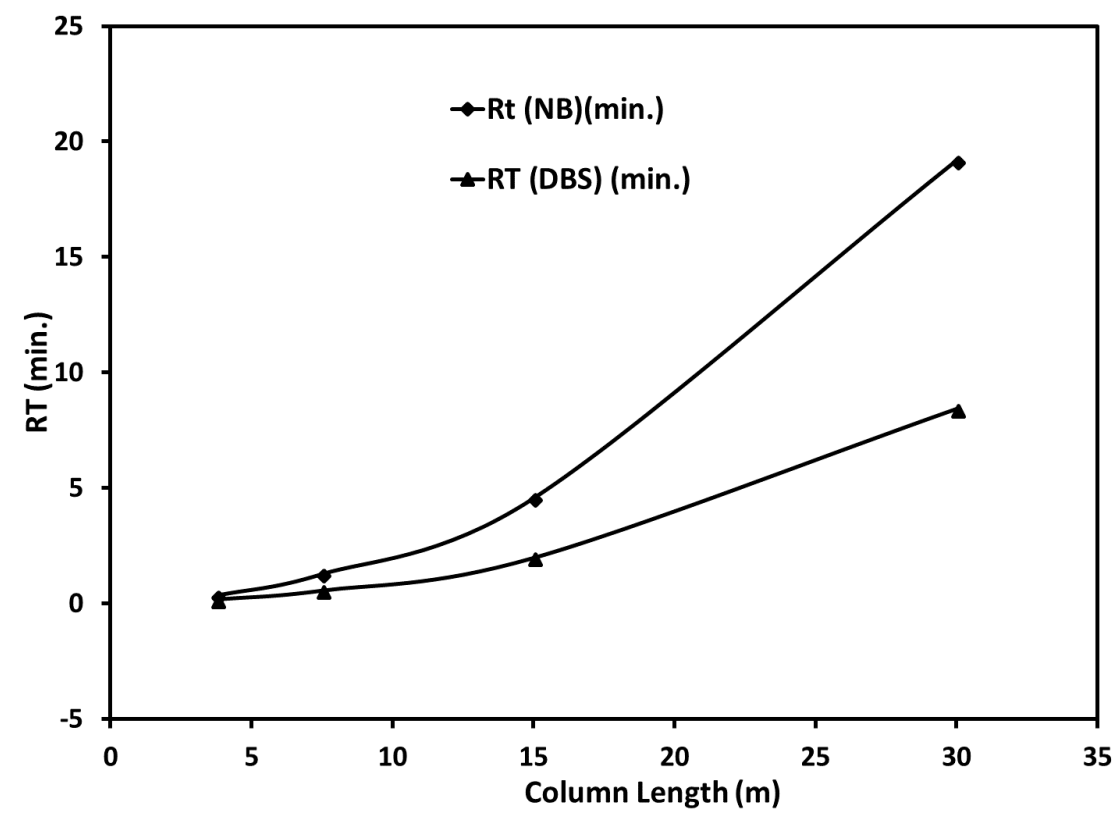

Fig 5. Effect of column length on Retention time of NB \& DBS at $60^{\circ} \mathrm{C}$

The same mixture of seven compounds were also injected to the injector port and recorded the at different temperature. The resolution/retention time of the chromatogram was reduced with higher the temperature of the oven (Figure 6 ).

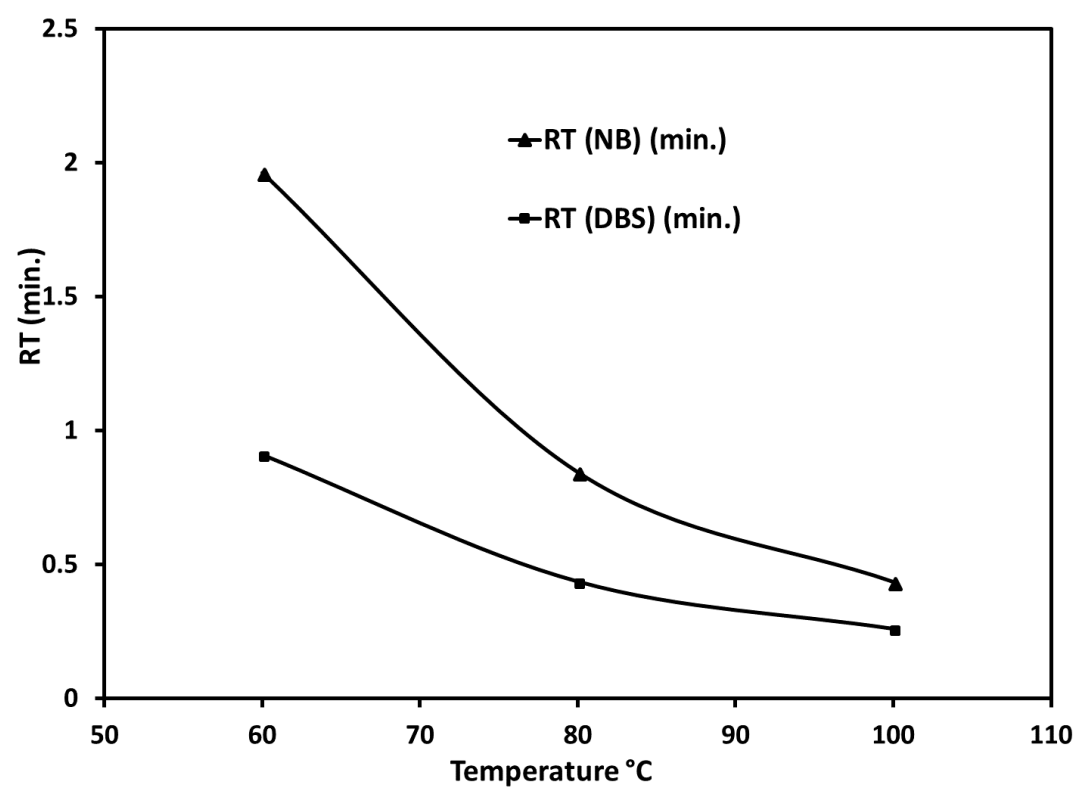

Fig 6. Effect of Temperature on Retention time of NB \& DBS

As per the analysis of the data it was observed that the resolution critically depends on three key parameter of GC i.e. flow rate of carrier, temperature of oven and length of capillary column ${ }^{(18,19)}$. It was also observed that the resolution of chromatogram of mixture of seven VOCs with FID at $60^{\circ} \mathrm{C}$ and with reduced length of column $(3.75 \mathrm{~m})$ was less resolved as compared to the SAW detector. 
As per the result it has been observed that the chromatogram with SAW detector is more resolved, less tailing and sharp peaks. However, the FID operates at higher temperature and it gives the less resolution and less sharp peaks (for same GC parameters) due to slow response time as compare to the SAW detector. Therefore, the following could be the possible reason for higher resolution of SAW detector as compared to the FID (Figure 7 ):

1. Higher sensitivity

2. Low active area on the surface of the uncoated SAW detector

3. Fast adsorption and desorption of eluted compounds

4. Low operating temperature of end part of column towards the SAW detector

Therefore, the SAW technology can be used for the fast analysis of VOCs.

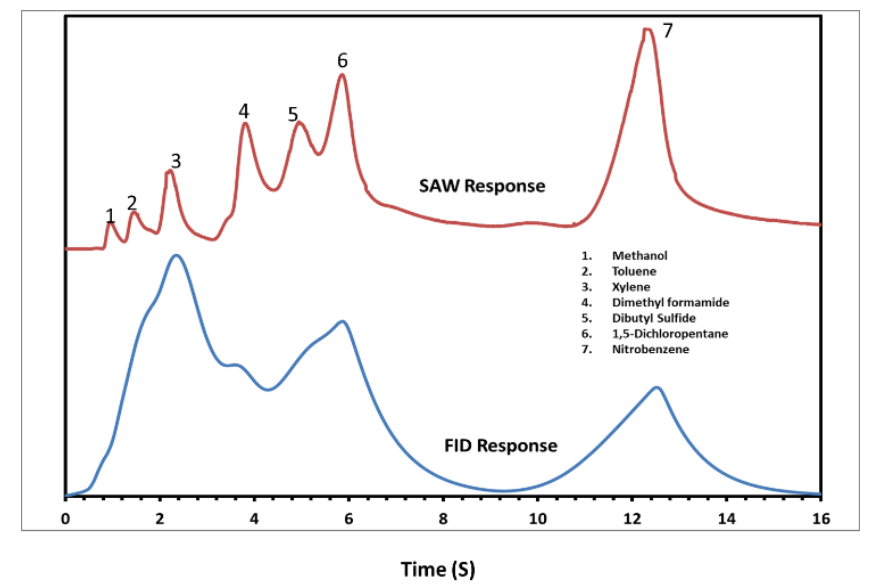

Fig 7. Response of FID and SAW detector with mixture of VOCs

\section{Conclusion}

We present the comparative study of resolution capability for Fast GC using FID and SAW detector. Both the detectors are capable of detecting of organic compounds in vapour phase. It is established from the findings of the work that SAW detector has better resolution as compared to FID and hence recommended for use in miniaturized sensor development. The SAW detector is also rugged, small size and low cost which makes it further most convenient choice of detector. The SAW detector does not require specific gases for its operation. As compared to the FID, SAW detector can resolve more number of compounds within specified time using small length of column.

\section{Acknowledgment}

Authors are thankful to Dr. Seema Vinayak, Director SSPL- DRDO, for her continuous support. Authors are also thankful to Pooja Rajput, Pallav Tripathi, Milap Singh, Sanjay and Chandani Kumari of SAW Group at SSPL for their support and patronage.

\section{References}

1) King WH. Piezoelectric Sorption Detector. Analytical Chemistry. 1964;36(9):1735-1739. Available from: https://dx.doi.org/10.1021/ac60215a012.

2) Wohltjen H, Dessy R. Surface acoustic wave probes for chemical analysis. II. Gas chromatography detector. Analytical Chemistry. 1979;51(9):1465-1470. Available from: https://dx.doi.org/10.1021/ac50045a025.

3) Zhou M, Lee J, Zhu H, Nidetz R, Kurabayashi K, Fan X. A fully automated portable gas chromatography system for sensitive and rapid quantification of volatile organic compounds in water. RSC Advances. 2016;6(55):49416-49424. Available from: https://dx.doi.org/10.1039/c6ra09131h.

4) Barbosa-Cornelio R, Cantor F, Coy-Barrera E, Rodríguez D. Tools in the investigation of volatile semiochemicals on insects: From sampling to statistical analysis. Insects. 2019;10(8):241. Available from: https://dx.doi.org/10.3390/insects10080241.

5) Bartelt-Hunt SL, Knappe DRU, Barlaz MA. A Review of Chemical Warfare Agent Simulants for the Study of Environmental Behavior. Critical Reviews in Environmental Science and Technology. 2008;38(2):112-136. Available from: https://dx.doi.org/10.1080/10643380701643650. 
6) Faricha A, Suwito S, Rivai M, Nanda MA, Purwanto D, Anhar RPR. Design of electronic nose system using gas chromatography principle and surface acoustic wave sensor. TELKOMNIKA (Telecommunication Computing Electronics and Control). 2018;16(4):1457. Available from: https://dx.doi.org/10. 12928/telkomnika.v16i4.7127.

7) Watson G, Staples E. SAW resonators as vapor sensors. 1990. Available from: https://doi.org/doi:10.1109/ultsym.1990.171375.

8) Watson G, Horton W, Staples E. Gas chromatography utilizing SAW sensors. 1991.

9) Staples EJ, Matsuda T, Viswanathan S. Real time environmental screening of air, water and soil matrices using a novel field portable GC / SAW system. In: and others, editor. Asia Pacific Conf. 1998;p. 8-10.

10) Witkiewicz Z. GC Analysis Encyclopedia of Chromatography, Third Edition Chemical Warfare Agents : GC Analysis. 2009. Available from: https: //doi.org/10.1081/E-ECHR3-120043106.

11) Detectors for the Gas Chromatographic Determination of Impurities. J Chromatogr Libr. 1991;49:25-34.

12) Slobodnik AJ. Surface acoustic waves and SAW materials. In: and others, editor. Proceedings of the IEEE;vol. 64. Institute of Electrical and Electronics Engineers (IEEE). 1976;p. 581-595. Available from: https://dx.doi.org/10.1109/proc.1976.10180.

13) Mittal U, Islam T, Nimal AT, Sharma MU. Fabrication of high frequency surface acoustic wave (SAW) devices for real time detection of highly toxic chemical vapors. International Journal on Smart Sensing and Intelligent Systems. 2015;8(3):1601-1623. Available from: https://dx.doi.org/10.21307/ijssis-2017-821.

14) Fahim M, Mittal U, Kumar J, Nimal AT, Sharma MU. Single chip readout electronics for SAW based gas sensor systems. Proc IEEE Sensors. 2017;p. 1-3. Available from: https://doi.org/10.1109/ICSENS.2017.8233886.

15) Liu J, Lu Y. Response mechanism for surface acoustic wave gas sensors based on surface-adsorption. Sensors (Switzerland). 2014;14:6844-6853. Available from: https://doi.org/10.3390/s140406844.

16) Dewulf J, Langenhove HV, Wittmann G. Analysis of volatile organic compounds using gas chromatography. TrAC Trends in Analytical Chemistry. 2002;21(9-10):637-646. Available from: https://dx.doi.org/10.1016/s0165-9936(02)00804-x.

17) Zhu X, Sun J, Ning Z, Zhang Y, Liu J. High performance mini-gas chromatography-flame ionization detector system based on micro gas chromatography column. Rev Sci Instrum. 2016;87. Available from: https://doi.org/10.1063/1.4946767.

18) De ROS. Effect of carrier gas flow rate and column temperature on the suitability parameters of gc-ecd for sfe6 measurement. 2019.

19) Giddings JC. Optimum conditions for separation in gas chromatography. Analytical Chemistry. 1960;32(12):1707-1711. Available from: https: //dx.doi.org/10.1021/ac60168a050. 